\title{
Variants at DGKB/TMEM195, ADRA2A, GLIS3 and $C 2 C D 4 B$ loci are associated with reduced glucose-stimulated beta cell function in middle-aged Danish people
}

\author{
T. W. Boesgaard • N. Grarup • T. Jørgensen • K. Borch-Johnsen • \\ Meta-Analysis of Glucose and Insulin-Related Trait \\ Consortium (MAGIC) • T. Hansen • O. Pedersen
}

Received: 15 December 2009/Accepted: 15 March 2010/Published online: 26 April 2010

(C) Springer-Verlag 2010

\begin{abstract}
Aims/hypothesis A meta-analysis of 21 genome-wide association studies identified 11 novel genetic loci implicated in fasting glucose homeostasis. We aimed to evaluate the impact of these variants on insulin release and insulin sensitivity estimated from OGTTs.

Methods Eleven variants in or near DGKB/TMEM195, ADCY5, MADD, ADRA2A, FADS1, CRY2, SLC2A2, GLIS3, $P R O X 1, C 2 C D 4 B$ and $I G F 1$ were genotyped in 6,784 middle-aged participants of the population-based Inter99 cohort. Association studies of quantitative estimates of insulin release and insulin sensitivity were performed in
\end{abstract}

T. W. Boesgaard and N. Grarup contributed equally to this work.

Electronic supplementary material The online version of this article (doi:10.1007/s00125-010-1753-5) contains supplementary material, which is available to authorised users. This includes a list of members of MAGIC.

T. W. Boesgaard $\cdot$ N. Grarup $(\bowtie) \cdot$ T. Hansen $\cdot$ O. Pedersen Hagedorn Research Institute,

Niels Steensens Vej 2,

2820 Gentofte, Denmark

e-mail: ngrp@hagedorn.dk

T. Jørgensen

Research Centre for Prevention and Health,

Glostrup University Hospital,

Glostrup, Denmark

T. Jørgensen

Faculty of Health Sciences, University of Copenhagen,

Copenhagen, Denmark

K. Borch-Johnsen

Steno Diabetes Center,

Copenhagen, Denmark
5,722 non-diabetic Danish participants on whom an OGTT was performed.

Results Assuming an additive genetic model, carriers of the alleles increasing fasting glucose in DGKB/TMEM195, $A D R A 2 A$, GLIS3 and $C 2 C D 4 B$ showed decreased glucosestimulated insulin release as assessed by the BIGTT-acute insulin response index $(2.7-3.5 \% ; p<0.005$ for all) and by corrected insulin response $(2.8-5.9 \% ; p<0.03$ for all). In addition, the $P R O X 1$ glucose-raising allele showed a $2.9 \%$ decreased corrected insulin response $(p=0.03)$, while the hyperglycaemic allele of variants in or near $A D R A 2 A$, FADS1, CRY2 and $C 2 C D 4 B$ were associated with a $2.6 \%$ to

K. Borch-Johnsen · O. Pedersen

Faculty of Health Sciences, University of Aarhus,

Aarhus, Denmark

T. Hansen

Faculty of Health Sciences, University of Southern Denmark, Odense, Denmark

O. Pedersen

Institute of Biomedical Science, Faculty of Health Sciences,

University of Copenhagen,

Copenhagen, Denmark 
$9.3 \%$ decrease in one or both of two different OGTT-based disposition indices $(p<0.02$ for all). After correction for multiple testing, variants in the DGKB/TMEM195, $A D R A 2 A, G L I S 3$ and $C 2 C D 4 B$ loci were associated with estimates of beta cell function.

Conclusions/interpretation We found that the lead variants at the DGKB/TMEM195, ADRA2A, GLIS3 and C2CD4B loci were associated with decreased glucose-stimulated insulin response. This association underlines the importance of pancreatic beta cell dysfunction in the genetic predisposition to hyperglycaemia and type 2 diabetes.

Keywords Association study B Beta cell dysfunction . Beta cell function - Genetic epidemiology · Glucose homeostasis · Glucose-stimulated insulin release .

Type 2 diabetes

\begin{tabular}{ll}
\multicolumn{2}{l}{ Abbreviations } \\
AIR & Acute insulin release \\
CIR & Corrected insulin response \\
DI & Disposition index \\
GWAS & Genome-wide association study \\
HOMA-B & HOMA of beta cell function \\
HOMA-IR & HOMA of insulin resistance \\
$S_{\text {I }}$ & Sensitivity index \\
SNP & Single nucleotide polymorphism
\end{tabular}

\section{Introduction}

For years it has been well known that genetic factors are crucially important for the development of type 2 diabetes. So far, genome-wide association studies (GWAS) and subsequent meta-analysis have identified 23 validated risk loci influencing risk of type 2 diabetes [1-3]. While many common gene variants have shown an influence on estimates of beta cell function, only few variants have an impact on insulin resistance [2, 4-7].

Besides evidence from association analysis of dichotomous phenotypes such as type 2 diabetes, novel knowledge of the genetic complexity of glucose regulation and homeostasis has come from GWAS of quantitative glycaemic traits. Hyperglycaemia in the fasting state is a major criterion in the definition of type 2 diabetes [8]. Type 2 diabetes is a risk factor for development of cardiovascular disease, but increased fasting glucose at non-diabetic levels also increases risk of cardiovascular events [9]. Several loci have been shown to influence this variable [10-17]. Recently, the international Meta-Analysis of Glucose and Insulin-related Trait Consortium (MAGIC) collaboration engaged in a large meta-analysis of data from 21 GWAS and identified novel loci influencing fasting glucose homeostasis. The initial discovery stage included 46,186 individuals. After replication in up to 76,558 individuals, 16 loci, ten of them novel, were found to be associated with fasting plasma glucose and one novel locus with fasting serum insulin at a genome-wide significant level [18]. Interestingly, variants at GCK, GCKR, DGKB/TMEM195, $A D C Y 5$ and PROXI loci showed novel association with type 2 diabetes at a genome-wide significance level in case-control studies of up to 127,677 individuals [18]. These five loci are thus part of the growing list of common type 2 diabetes susceptibility loci [18]. Also, several variants showed robust association with the HOMA index of beta cell function (HOMA-B), a surrogate measure of beta cell function in the fasting state.

While fasting levels of glucose and the derived HOMA-B index may depict important processes in the regulation of basal glycaemia, they are not informative with regard to complex dynamic postprandial glucose regulation $[19,20]$. We therefore evaluated possible associations with estimates of insulin release and insulin sensitivity based on outcomes of OGTT, with a view to further describing the relationship between these variants and elements of glucose homeostasis. We investigated the 11 novel loci implicated in fasting glucose homeostasis in 5,722 non-diabetic individuals of the population-based Inter99 study on whom an OGTT had been performed. The remaining six loci have previously been investigated in this population [10, 12, 21-23].

\section{Methods}

Participants The Inter99 cohort is a population-based, randomised, non-pharmacological intervention study for the prevention of ischaemic heart disease, conducted on 6,784 middle-aged participants at the Research Centre for Prevention and Health in Glostrup, Copenhagen (ClinicalTrials.gov: NCT00289237) [24]. An OGTT was performed with measurement of plasma glucose and serum insulin at fasting, and at 30 and $120 \mathrm{~min}$ after glucose intake. Subsequently, 6,094 participants of Danish nationality and with available DNA were classified as having normal glucose tolerance $(n=4,525)$, impaired fasting glycaemia $(n=504)$, impaired glucose tolerance $(n=693)$, screendetected and treatment-naive diabetes $(n=253)$, or previously diagnosed diabetes $(n=119)$ according to WHO 1999 criteria [8]. The analysis of quantitative diabetes-related phenotypes was performed in 5,722 non-diabetic participants. Detailed characteristics of Inter99 are presented in Table 1.

Informed written consent was obtained from all individuals before participation. The study was approved by the Ethical Committee of Copenhagen County and conducted in accordance with the principles of the Helsinki Declara- 
Table 1 Quantitative metabolic traits in the population-based Inter99 cohort
Data are median $(25-75 \%$ range) or mean \pm SD. DI 1 was calculated as BIGTT-AIR $\times$ BIGTT- $S_{\text {I }}$; DI 2 was calculated as CIR $\times 1 /$ HOMA-IR

${ }^{\mathrm{a}}$ With available DNA and glucose tolerance determined

\begin{tabular}{|c|c|c|}
\hline \multirow[t]{2}{*}{ Characteristics } & \multicolumn{2}{|l|}{ Inter99 participants } \\
\hline & Full cohort $^{\mathrm{a}}$ & Non-diabetic individuals ${ }^{\mathrm{a}}$ \\
\hline$n$ & 6,094 & 5,722 \\
\hline Men $(n)$ & 3,036 & 2,814 \\
\hline Women $(n)$ & 3,058 & 2,908 \\
\hline Age (years) & $46 \pm 8$ & $46 \pm 8$ \\
\hline BMI $\left(\mathrm{kg} / \mathrm{m}^{2}\right)$ & $26.3 \pm 4.6$ & $26 \pm 4.4$ \\
\hline Waist circumference $(\mathrm{cm})$ & $86.7 \pm 13$ & $85.9 \pm 13$ \\
\hline Fasting plasma glucose $(\mathrm{mmol} / \mathrm{l})$ & $5.6 \pm 1.1$ & $5.5 \pm 0.5$ \\
\hline 30 min plasma glucose $(\mathrm{mmol} / \mathrm{l})$ & $8.7 \pm 1.9$ & $8.6 \pm 1.7$ \\
\hline $120 \mathrm{~min}$ plasma glucose $(\mathrm{mmol} / \mathrm{l})$ & $6.2 \pm 2.2$ & $6.0 \pm 1.5$ \\
\hline Fasting serum insulin (pmol/l) & $35(24-52)$ & $34(23-49)$ \\
\hline 30 min serum insulin $(\mathrm{pmol} / \mathrm{l})$ & $246(175-355)$ & $246(176-355)$ \\
\hline 120 min serum insulin $(\mathrm{pmol} / \mathrm{l})$ & $157(96-257)$ & $153(94-246)$ \\
\hline $\mathrm{HbA}_{1 \mathrm{c}}(\%)$ & $5.8(5.6-6.1)$ & $5.8(5.5-6.1)$ \\
\hline Corrected insulin response & $92.9(57-152)$ & $96.1(59.8-156)$ \\
\hline BIGTT-AIR & $1,630(1,280-2,080)$ & $1,640(1,300-2,090)$ \\
\hline HOMA-IR & $1.21(0.82-1.90)$ & $1.16(0.80-1.76)$ \\
\hline BIGTT- $S_{\mathrm{I}}$ & $9.18 \pm 4.10$ & $9.44 \pm 3.90$ \\
\hline DI 1 & $14,800(10,300-19,800)$ & $15,200(10,900-20,000)$ \\
\hline DI 2 & $74.8(41.6-137)$ & $79.2(44.9-141)$ \\
\hline
\end{tabular}

tion II. Biochemical and anthropometric measures were obtained as previously detailed [25].

Indices of insulin release and insulin sensitivity Oral glucose-stimulated insulin release was reported as serum insulin at $30 \mathrm{~min}$ during the OGTT, the BIGTT-acute insulin response (AIR) index and the corrected insulin response (CIR). Insulin sensitivity was reported as the OGTT-derived BIGTT sensitivity index $\left(S_{\mathrm{I}}\right)$. CIR was calculated as: $\left(\left[\right.\right.$ serum insulin $\left.\left._{30 \min }\left\{\mathrm{pmol}_{/} / 1\right\} / 6.945\right] \times 100\right) /$ (plasma glucose $30 \mathrm{~min}\{\mathrm{mmol} / 1\} \times\left[\right.$ plasma glucose $\operatorname{momin}_{30 \mathrm{mmol}}\{\mathrm{m}$ $1\}-3.89]$ ) [26]. The BIGTT indices apply information on sex and BMI, combined with plasma glucose and serum insulin during an OGTT, to provide indices for AIR and $S_{\mathrm{I}}$, and were calculated as reported [27]. In order to construct OGTT-based disposition indices (DIs) we multiplied BIGTT-AIR with BIGTT-S $S_{\text {I }}$ (DI 1) and CIR with the reciprocal of HOMA of insulin resistance (HOMA-IR) (DI 2). The DI is an estimate of the ability of the pancreatic beta cell to respond appropriately to concomitant levels of insulin sensitivity.

Genotyping We genotyped 11 gene variants by KASPar SNP Genotyping system (KBiosciences, Hoddesdon, UK). All genotyping success rates were above $96 \%$ with a mismatch rate below $0.5 \%$ in 591 pairs genotyped in duplicate. The distribution of genotypes for all variants were in Hardy-Weinberg equilibrium in Inter99 $(p>0.05)$, except for $\mathrm{rs} 21911349$ and $\mathrm{rs} 11920090(p=0.01$ and $p=$ 0.008 , respectively).

Statistical analysis General linear statistical methodology was used to test quantitative traits in relation to genotype, applying additive models adjusting for the effect of age (BIGTT- $S_{\text {I }}$ and BIGTT-AIR) or age and sex (all other traits). Values of serum insulin and derived indices (except BIGTT- $S_{\mathrm{I}}$ ) were logarithmically transformed prior to analysis. The multivariate method, Hotelling's $T^{2}$, was applied to test the simultaneous effect of genotype on BIGTT- $S_{\text {I }}$ and BIGTT-AIR. A $p$ value of less than 0.05 was considered significant. Correlation between quantitative metabolic traits independent of genotype was performed by Pearson correlation. Non-normally distributed traits were normalised by log-transformation. For the association of gene variants with fasting plasma glucose we calculated the proportion that could be accounted for by association with insulin release or insulin sensitivity by dividing the expected effect with the observed effect. The expected effect of gene variants on fasting plasma glucose, given the association with insulin release (BIGTT-AIR) or insulin sensitivity (BIGTT- $S_{\mathrm{I}}$ ), was calculated by multiplying the single nucleotide polymorphism (SNP) effect on insulin release by the epidemiological effect between insulin release and fasting plasma glucose. This triangulation approach has been described previously [28]. The proportion of variance explained by genetic variants was assessed 
by the partial $R^{2}$ from a linear regression model including main effects of all selected variants. Analyses were performed using RGui, version 2.10.0 (www.r-project.org).

Statistical power Statistical power was calculated as previously reported [25]. Depending on allele frequency (range $11-49 \%$ ), we had $80 \%$ statistical power at a $5 \%$ significance level to detect an allele-dependent difference of $5.3 \%$ to $8.4 \%$ of a standard deviation corresponding to a $2.2 \%$ to $3.5 \%$ difference in BIGTT-AIR, a $3.8 \%$ to $6.0 \%$ difference in CIR and an absolute 0.21 to 0.33 difference in BIGTT- $S_{\text {I }}$.

\section{Results}

We genotyped the following 11 variants in the Danish population-based Inter99 cohort (Table 1): $D G K B /$ TMEM195 rs2191349, ADCY5 rs11708067, MADD rs7944584, ADRA2A rs10885122, FADS1 rs174550, CRY2 rs11605924, SLC2A2 rs11920090, GLIS3 rs7034200, PROXI rs340874, C2CD4B rs11071657 and IGFI rs35767. Results in Inter99 for traits reflecting fasting glucose homeostasis (plasma glucose, serum insulin, HOMA-IR and HOMA-B) have been previously reported [18], but are presented for comparison in Table 2 and Electronic supplementary materials (ESM) Table 1. Inter99 association results for six known fasting glucose loci have been published previously [10, 12, 21-23]. In 5,722 nondiabetic Inter99 participants we tested the above-named 11 variants for association with quantitative OGTT-based estimates of beta cell function and insulin sensitivity (Table 2). Carriers of the DGKB/TMEM195 rs2191349 glucose-raising T-allele showed decreased glucosestimulated insulin release as assessed by serum insulin at $30 \mathrm{~min}(2.7 \%$ [95\% CI 0.6-4.7], $p=0.01)$, BIGTT-AIR (2.7\% [1.1-4.2], $p=0.0009)$ and CIR (2.8\% [0.2-5.5\%], $p=$ 0.03 ; Table 2). Likewise, carriers of the glucose-raising Gallele of ADRA2A rs10885122 showed a consistent decreased insulin response after oral glucose ingestion evaluated by CIR (5.9\% [1.8-10\%], $p=0.005)$, BIGTTAIR (3.5\% [1.1-6.0\%], $p=0.005)$ and two different disposition indices (DI $14.2 \%$ [1.2-7.1\%], $p=0.005$, DI 2 9.3\% [4.6-14\%], $p=0.0001$; Table 2). Also, carriers of the glucose-raising A-allele of $C 2 C D 4 B$ rs 11071657 had a $4.3 \%(1.6-7.0 \%, p=0.002)$ decreased insulin release as assessed by the CIR index, consistent with a $3.2 \%$ (1.5$4.8 \%, p=0.0002$ ) decreased BIGTT-AIR index and a $3.1 \%$ $(1.1-5.0 \%, p=0.002)$ decreased DI (Table 2). Similarly, carriers of the glucose-raising A-allele of GLIS3 rs7034200 had a decreased glucose-stimulated insulin release assessed by BIGTT-AIR (3.1\% [1.4-4.7\%], $p=0.0002)$. The $C R Y 2$ rs11605924 glucose-raising A-allele was associated with a
2.6 to $3.8 \%$ decrease in two different disposition indices $(p<0.02)$, while the diabetes-related PROX1 rs340874 Callele showed a $2.7 \%(0.6-4.8 \%, p=0.01)$ decreased serum insulin at 30 min during OGTT and $2.9 \%$ decreased CIR $(0.3-5.5 \%, p=0.03)$. Also, association with a single estimate of glucose-induced insulin response was observed for $M A D D$ rs7944584 (DI $23.9 \%$ [0.4-7.4\%], $p=0.03$ ), FADS1 rs 174550 (DI $1-2.5 \%$ [ -4.4 to $-0.51 \%], p=0.01$ ) and SLC2A2 (CIR 4.3\% [0.6-8.1\%], $p=0.02$ ). Besides association of the $C R Y 2$ variant $(p=0.02)$, none of the examined variants was associated with the BIGTT- $S_{\text {I }}$ estimate of insulin sensitivity. All results were virtually unchanged in models including BMI as an explanatory variable or when analysing a subset of participants with normal glucose tolerance (data not shown).

Given this complex pattern of association we determined the pairwise correlation between the investigated OGTTderived traits and traits reflecting fasting glucose homeostasis in all non-diabetic participants of Inter99 (ESM Table 2). These analyses showed modest correlation between various OGTT-based indices of glucose-stimulated insulin release, accounting for some of the discrepancies in observed associations.

The relationship between concomitant insulin sensitivity and insulin release from the pancreatic beta cell is described by a hyperbola [29]. To illustrate the genotyperelated relationship between estimates of insulin sensitivity and insulin release, we plotted two-dimensional standard error ellipses of the genotype-specific means of BIGTT- $S_{\text {I }}$ and BIGTT-AIR (Fig. 1). Variants at $D G K B /$ TMEM195, ADRA2A, GLIS3 and C2CD4B loci showed a distinct pattern consistent with a main effect on BIGTTAIR, whereas the two-dimensional impact of variants in CRY2, PROX1 and FADS1 loci did not support an effect on glucose-stimulated insulin release. In this bivariate analysis the CRY2 variant was associated with BIGTTAIR and BIGTT- $S_{\text {I }}$, underlining the association with the BIGTT- $S_{\text {I }}$ estimate of insulin sensitivity in univariate analysis (Table 2).

To assess the proportion of the association with fasting plasma glucose (Table 2) that could be attributed to insulin release or insulin sensitivity, we calculated the expected effect size for association between a SNP and fasting glucose given the SNP association with the BIGTT-AIR or BIGTT- $S_{\text {I }}$ estimates of insulin release and sensitivity, and the SNP-independent association between BIGTT-AIR or BIGTT- $S_{\text {I }}$ and fasting glucose. We then compared the expected effect with the observed effect on fasting glucose to assess the proportion of association possibly mediated by insulin release or sensitivity (ESM Table 3). These calculations were done for the seven variants associated with fasting plasma glucose in Inter99. For the four loci strongly associated with insulin release in Inter99 (DGKB/ 


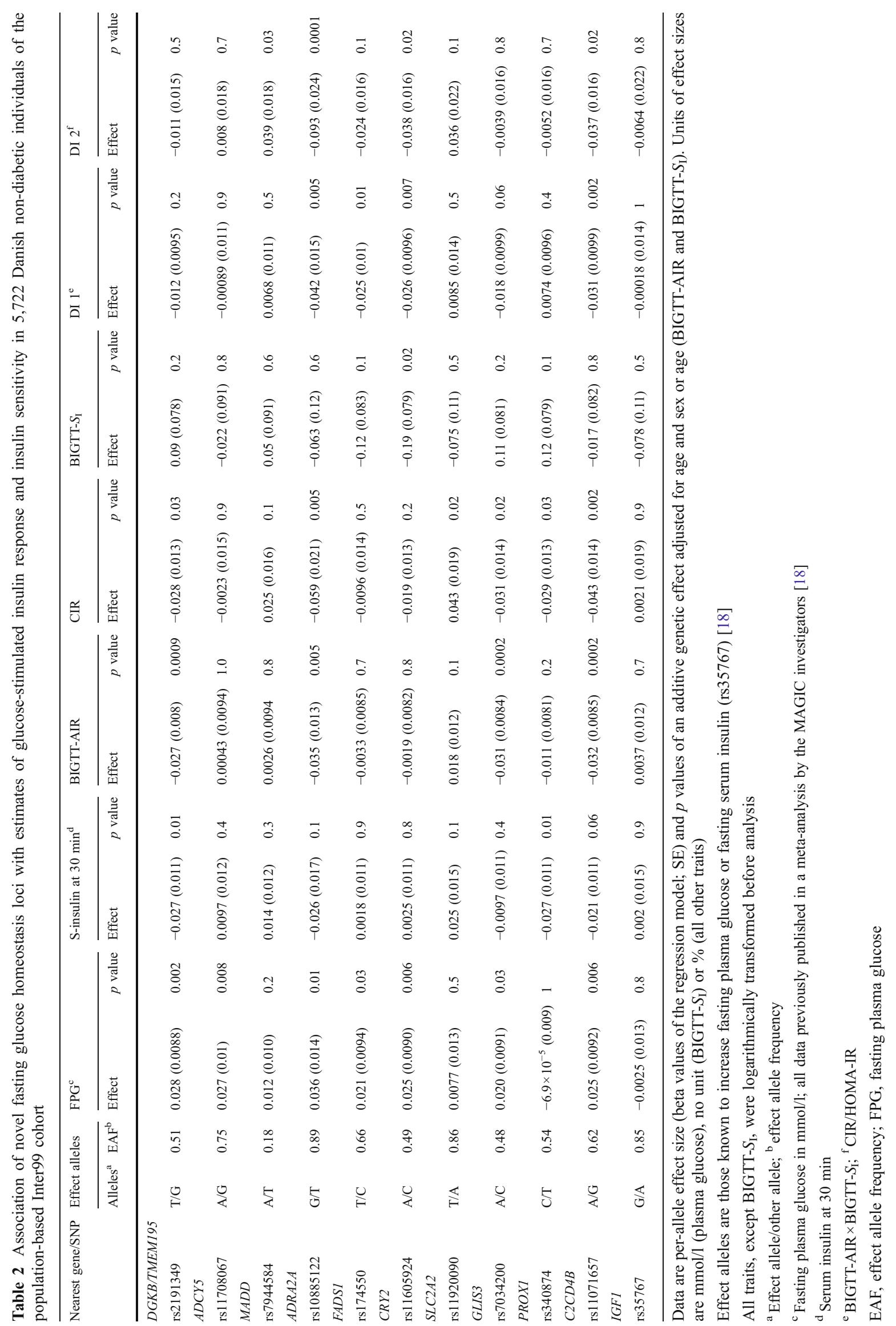



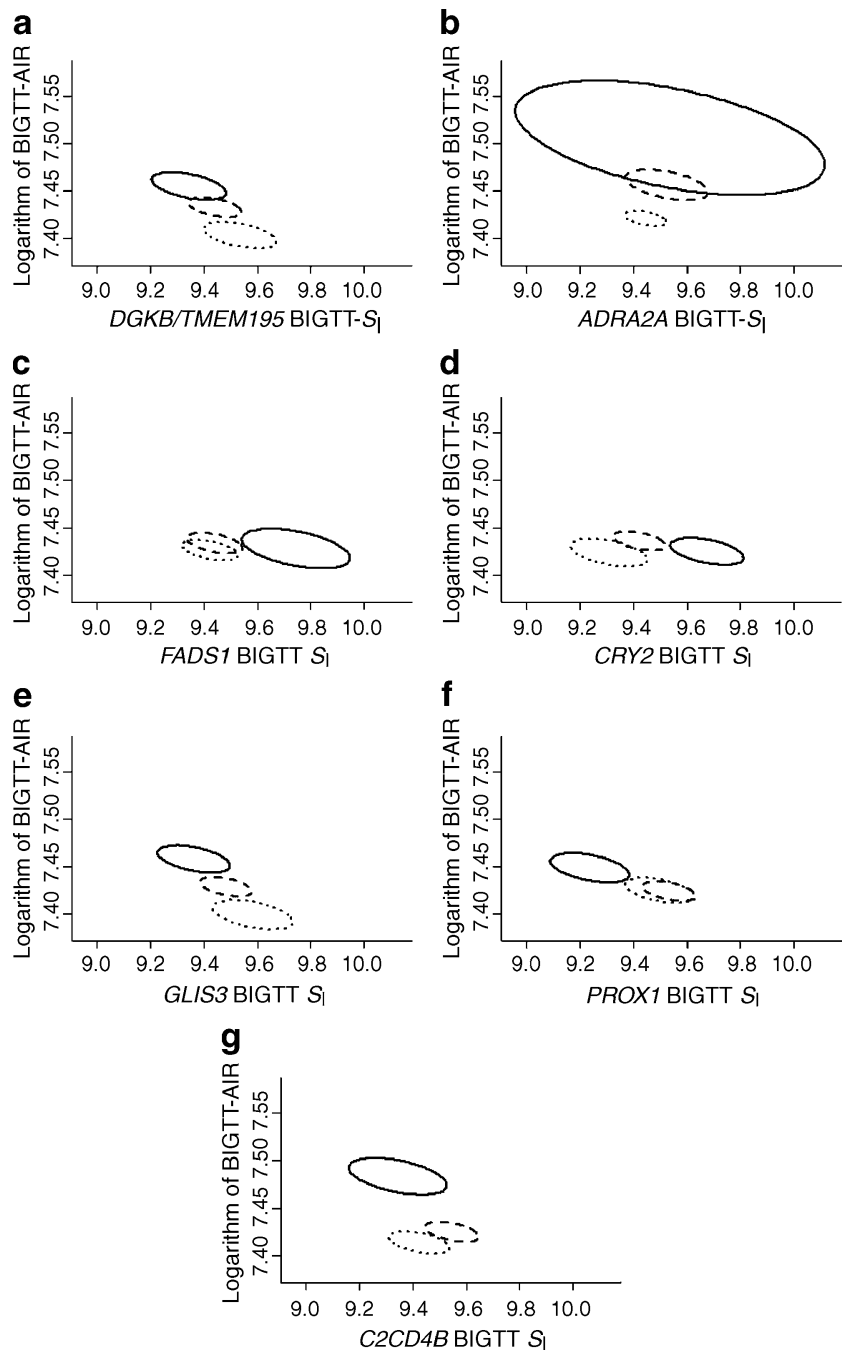

Fig. 1 Two-dimensional SEM of BIGTT- $S_{\text {I }}$ and logarithm of BIGTTAIR stratified according to the genotype of (a) DGKB/TMEM195 rs2191349, (b) ADRA2A rs10885122, (c) FADS1 rs174550, (d) CRY2 rs11605924, (e) GLIS3 rs7034200, (f) PROX1 rs340874 and (g) $C 2 C D 4 B$ rs 11071657 in 5,722 non-diabetic participants of the Inter99 cohort. SE ellipses around the mean of each genotype level were constructed assuming bivariate normal distribution. The multivariate method, Hotelling $T^{2}$, was applied to test the simultaneous effect of genotype on the two traits of insulin release and insulin sensitivity assuming an additive model with age as a covariate. Continuous line, SE for homozygous reference allele carriers; dashed line, SE for heterozygous carriers; dotted line, SE for homozygous glucose-raising allele carriers. a $p=0.005 ;$ (b) $p=0.001 ;$ (c) $p=0.2$; (d) $p=0.03$; (e) $p=$ $0.002 ;(\mathbf{f}) p=0.2 ;(\mathbf{g}) p=5 \times 10^{-4}$

TMEM195, ADRA2A, GLIS3 and C2CD4B), the proportion of effect on fasting glucose that could be ascribed to association with BIGTT-AIR ranged from $34 \%$ to $57 \%$. Also, the proportions of effect on fasting glucose at the FADS1 and CRY2 loci that were explained by association with insulin sensitivity (BIGTT- $S_{\mathrm{I}}$ ) were $39 \%$ and $48 \%$, respectively (ESM Table 3).

\section{Discussion}

Recent advances in the genetic exploration of traits related to fasting glucose homeostasis have revealed 17 loci associated with fasting plasma glucose or fasting serum insulin [18], of which six known loci have been previously reported [10, 12, 21-23]. In the current paper we aimed to evaluate the impact of variants at the 11 novel loci on OGTT-based insulin sensitivity and to estimate beta cell function by analysis of a range of measures of glucosestimulated insulin release in non-diabetic middle-aged individuals. The study demonstrates that of ten novel loci associated with increased fasting plasma glucose at least four (DGKB/TMEM195, ADRA2A, GLIS3 and C2CD4B), and possibly one additional locus ( $P R O X 1)$, are associated with reduced insulin response to oral glucose. These loci thus join the growing list of common variants with a modest effect on pancreatic beta cell function. We found that the lead variants in or near DGKB/TMEM195, $A D R A 2 A, C R Y 2, G L I S 3, P R O X 1$ and $C 2 C D 4 B$ were associated with two or more of five correlated estimates of insulin release, while variants in or near FADS1, SLC2A2 and $M A D D$ were associated with a single insulin release measure. With the exception of probably sporadic associations with $S L C 2 A 2$ and $M A D D$, all effects showed a decrease in glucose-stimulated insulin release in carriers of the allele that also increased fasting glucose in the previous combined report [18]. However, two-dimensional plots of insulin sensitivity in relation to insulin release did not support the impact of CRY2 and FADS1 variants on glucose-stimulated insulin release. In line with univariate analysis, two-dimensional data for $C R Y 2$ pointed to a possible impact on insulin sensitivity. Yet, the MAGIC study did not show an effect on HOMA-IR in more than 35,000 individuals, underlining the uncertainty of the present association [18].

The IGF1 variant previously associated with increased fasting insulin levels and increased HOMA-IR did not associate with an OGTT-based insulin $S_{\mathrm{I}}$ in the present study, highlighting the difficulties in establishing genetic variants that influence insulin sensitivity. Besides lack of statistical power, these difficulties could be explained by the relatively poor phenotypic estimation of insulin resistance in most datasets. Also, insulin resistance variants are probably more prone to interaction with correlated phenotypes or environmental factors, such as obesity, physical activity or dietary intake, thus obscuring association and replication in studies of genetic main effects [6].

Association of DGKB/TMEM195, ADRA2A, GLIS3, $P R O X 1$ and $C 2 C D 4 B$ loci with glucose-stimulated insulin release is consistent with genes in these loci being expressed in the pancreas and human islet cells [18]. However, the more exact molecular mechanisms of action 
cannot be inferred from the current data. Interestingly, $D G K B / T M E M 195$ rs2191341 and PROX1 rs340874 were associated with type 2 diabetes at genome-wide significance $\left(p<5 \times 10^{-8}\right)$ in the MAGIC publication, while the other three variants showing an effect on pancreatic beta cell function had limited impact on risk of type 2 diabetes in data from the MAGIC investigators (OR 1.03-1.04, $p=$ $0.001-0.02)$ [18]. In addition, when comparing the effect on insulin release of variants associated with type 2 diabetes in the MAGIC publication [18] with that of variants not associated with type 2 diabetes, we observed no clear distinction between these groups. Given these observations, there is no clear correlation between genetically impaired beta cell function in the general population and risk of type 2 diabetes. However, it is possible that lack of statistical power to obtain high confidence in association for these low-impact variants may have obscured the genuine coherence.

Our novel findings of glucose-stimulated insulin release are largely in agreement with the MAGIC paper [18], in which four of the ten fasting glucose variants examined here were also associated at genome-wide significance with basal beta cell function under non-stimulated conditions as estimated from the HOMA-B model. In our study of largescale genetic physiology, we applied OGTT to estimate the glucose-stimulated insulin response, a method that is superior to the HOMA-B model in providing a reliable surrogate measure of first-phase insulin release [19, 27]. Yet the correlation between different OGTT-based estimates of insulin release was not complete, as shown by results in ESM Table 2. Interestingly, HOMA-B correlated strongly with HOMA-IR and fasting serum insulin, and modestly with the CIR and BIGTT-AIR estimates of insulin release in Inter99. Therefore it is essential to investigate stimulated insulin release in order to substantiate a beta cell dysfunction as the pathogenic mechanism potentially leading to increased fasting glucose and type 2 diabetes. The importance of this statement is stressed by the observation that $A D R A 2 A$ rs10885122 was not associated with HOMA$\mathrm{B}$ at genome-wide significance in the MAGIC paper and did not replicate strongly $(p=0.03)$ in $\sim 60,000$ individuals [18]; yet the current data provide strong evidence of a postprandial insulin release defect. The functional and genetic impact of $A D R A 2 A$ on dynamic insulin secretion was also described in a recent paper showing reduced in vitro insulin secretion and impaired docking of insulin granule in carriers of variants in $A D R A 2 A$ [30]. Despite the application of OGTT-based estimates of beta cell function, we cannot rule out the possibility that the lack of consistent association with beta cell function for variants at $A D C Y 5$, $M A D D, F A D S 1, C R Y 2$ and $S L C 2 A 2$ in the present study is due to lack of statistical power. Interestingly, in up to 61,907 replication samples of the MAGIC study [18], variants in $M A D D, C R Y 2$ and $S L C 2 A 2$ that had otherwise been associated with HOMA-B did not convincingly replicate, a finding supported by the current data, which question the effect of these variants on pancreatic beta cell function. Also, the lack of consistent association of the $A D C Y 5$ variant with orally stimulated insulin release in the present report is in accordance with results of another report by the MAGIC investigators involving $\sim 19,000$ OGTTinvestigated individuals who were tested for the highly correlated $\left(r^{2}=0.85\right)$ rs2877716 variant [31].

In Inter99 we had $80 \%$ statistical power to detect an effect size of 0.05 to $0.08 \mathrm{SD}$ depending on allele frequency. For comparison, the effect on fasting plasma glucose in Inter99 ranged from $0 \mathrm{SD}(C 2 C D 4 B)$ to $0.07 \mathrm{SD}(A D R A 2 A)$, indicating that the Inter99 cohort is marginally underpowered to detect such low impacts. Also, we acknowledge that the present report applies a liberal statistical significance threshold. Performing conservative Bonferroni correction for 11 gene variants implies a corrected alpha of 0.0045 , leaving associations between glucose-stimulated insulin response and variants at $D G K B / T M E M 195, A D R A 2 A, G L I S 3$ and $C 2 C D 4 B$ loci significant at this threshold.

Interestingly, from a genetic viewpoint the major impact on risk of type 2 diabetes and fasting hyperglycaemia is through decreased function of the pancreatic beta cell. In triangulation analyses we estimated that for the four variants with an impact on beta cell function, around $30 \%$ to $60 \%$ of the effect on fasting plasma glucose could be accounted for by association with glucose-stimulated insulin release. These estimates suggest the existence of additional glucose-raising mechanisms that are independent of glucose-stimulated insulin release. Despite the high number of statistically highly associated variants discovered in the last few years, the explained proportion of the interindividual variation reported in Inter99 for the combination of all 33 validated type 2 diabetes and/or fasting plasma glucose gene variants is only $5.8 \%$ for fasting plasma glucose and $4.2 \%$ to $5.7 \%$ for different estimates of glucose-stimulated insulin release in non-diabetic Danish people (data not shown). These numbers should be interpreted in relation to heritability estimates of $30 \%$ to $40 \%$ and $60 \%$ to $80 \%$ for fasting glucose and first-phase insulin release, respectively [32], indicating that $15 \%$ to $20 \%$ and $5 \%$ to $10 \%$, respectively, of the genetic contribution to these traits has been accounted for. These estimates imply that a major part of the genetic contribution to these quantitative traits is yet to be unravelled.

In conclusion, in a large OGTT-investigated populationbased cohort we found that the lead variants at the $D G K B$ / TMEM195, ADRA2A, GLIS3 and C2CD4B loci were associated with decreased glucose-stimulated insulin response, underlining the importance of pancreatic beta cell dysfunction in genetic predisposition to hyperglycaemia and type 2 diabetes. 
Acknowledgements The authors wish to thank A. Forman, I.-L. Wantzin and M. Stendal for technical assistance, and A. L. Nielsen, G. Lademann and M. M. H. Kristensen for management assistance. The study was supported by grants from: the Lundbeck Foundation Centre of Applied Medical Genomics for Personalized Disease Prediction, Prevention and Care (LuCAMP), the Danish Health Research Council, the European Union (EXGENESIS, grant no. LSHM-CT-2004-005272), the Danish Diabetes Association, the Danish Council for Independent Research (Medical Sciences) and Novo Nordisk. The Inter99 was initiated by T. Jørgensen (Principal Investigator), K. Borch-Johnsen (co-Principal Investigator), H. Ibsen and T. F. Thomsen. The steering committee comprises the former two and C. Pisinger. The study was financially supported by research grants from the Danish Research Council, the Danish Centre for Health Technology Assessment, Novo Nordisk, Research Foundation of Copenhagen County, Ministry of Internal Affaires and Health, the Danish Heart Foundation, the Danish Pharmaceutical Association, the Augustinus Foundation, the Ib Henriksen Foundation, the Becket Foundation and the Danish Diabetes Association.

Duality of interest K. Borch-Johnsen is director of the Steno Diabetes Center, a hospital integrated into the Danish National Health Care Service and owned by Novo Nordisk, in which K. BorchJohnsen holds employee shares. All other authors declare that there is no duality of interest associated with this manuscript.

\section{References}

1. McCarthy MI, Zeggini E (2009) Genome-wide association studies in type 2 diabetes. Curr Diab Rep 9:164-171

2. Rung J, Cauchi S, Albrechtsen A et al (2009) Genetic variant near IRS1 is associated with type 2 diabetes, insulin resistance and hyperinsulinemia. Nat Genet 41:1110-1115

3. Kong A, Steinthorsdottir V, Masson G et al (2009) Parental origin of sequence variants associated with complex diseases. Nature 462:868-874

4. Deeb SS, Fajas L, Nemoto M et al (1998) A Pro12Ala substitution in PPARgamma2 associated with decreased receptor activity, lower body mass index and improved insulin sensitivity. Nat Genet 20:284-287

5. Boesgaard TW, Gjesing AP, Grarup N et al (2009) Variant near ADAMTS9 known to associate with type 2 diabetes is related to insulin resistance in offspring of type 2 diabetes patientsEUGENE2 Study. PLoS ONE 4:e7236

6. Florez JC (2008) Newly identified loci highlight beta cell dysfunction as a key cause of type 2 diabetes: where are the insulin resistance genes? Diabetologia 51:1100-1110

7. Staiger H, Machicao F, Fritsche A, Haring HU (2009) Pathomechanisms of type 2 diabetes genes. Endocr Rev 30:557-585

8. World Health Organization Study Group (1999) Definition, diagnosis and classification of diabetes mellitus and its complications. Part 1: diagnosis and classification of diabetes mellitus. Technical Report Series WHO/NCD/NCS/99.2 ed. World Health Organization, Geneva

9. Coutinho M, Gerstein HC, Wang Y, Yusuf S (1999) The relationship between glucose and incident cardiovascular events. A metaregression analysis of published data from 20 studies of 95 , 783 individuals followed for 12.4 years. Diabetes Care 22:233-240

10. Rose CS, Ek J, Urhammer SA et al (2005) A $-30 \mathrm{G}>\mathrm{A}$ polymorphism of the beta-cell-specific glucokinase promoter associates with hyperglycemia in the general population of whites. Diabetes 54:3026-3031

11. Weedon MN, Clark VJ, Qian Y et al (2006) A common haplotype of the glucokinase gene alters fasting glucose and birth weight: association in six studies and population-genetics analyses. Am J Hum Genet 79:991-1001

12. Sparsø T, Andersen G, Nielsen T et al (2008) The GCKR rs780094 polymorphism is associated with elevated fasting serum triacylglycerol, reduced fasting and OGTT-related insulinaemia, and reduced risk of type 2 diabetes. Diabetologia 51:70-75

13. Bouatia-Naji N, Rocheleau G, Van-Lommel L et al (2008) A polymorphism within the G6PC2 gene is associated with fasting plasma glucose levels. Science 320:1085-1088

14. Chen WM, Erdos MR, Jackson AU et al (2008) Variations in the G6PC2/ABCB11 genomic region are associated with fasting glucose levels. J Clin Invest 118:2620-2628

15. Bouatia-Naji N, Bonnefond A, Cavalcanti-Proença C et al (2009) A variant near MTNR1B is associated with increased fasting plasma glucose levels and type 2 diabetes risk. Nat Genet 41:89-94

16. Lyssenko V, Nagorny CL, Erdos MR et al (2009) Common variant in MTNR1B associated with increased risk of type 2 diabetes and impaired early insulin secretion. Nat Genet 41:82-88

17. Prokopenko I, Langenberg C, Florez JC et al (2009) Variants in MTNR1B influence fasting glucose levels. Nat Genet 41:77-81

18. Dupuis J, Langenberg C, Prokopenko I et al (2010) New genetic loci implicated in fasting glucose homeostasis and their impact on type 2 diabetes risk. Nat Genet 42:105-116

19. Cobelli C, Toffolo GM, Dalla-Man C et al (2007) Assessment of beta-cell function in humans, simultaneously with insulin sensitivity and hepatic extraction, from intravenous and oral glucose tests. Am J Physiol Endocrinol Metab 293:E1-E15

20. Matthews DR, Hosker JP, Rudenski AS, Naylor BA, Treacher DF, Turner RC (1985) Homeostasis model assessment: insulin resistance and beta-cell function from fasting plasma glucose and insulin concentrations in man. Diabetologia 28:412-419

21. Sparsø T, Bonnefond A, Andersson E et al (2009) The G-allele of intronic rs10830963 in MTNR1B confers increased risk of impaired fasting glycemia and type 2 diabetes through an impaired glucose-stimulated insulin release: studies involving 19,605 Europeans. Diabetes 58:1450-1456

22. Steinthorsdottir V, Thorleifsson G, Reynisdottir I et al (2007) A variant in CDKAL1 influences insulin response and risk of type 2 diabetes. Nat Genet 39:770-775

23. Rose CS, Grarup N, Krarup NT et al (2009) A variant in the G6PC2/ABCB11 locus is associated with increased fasting plasma glucose, increased basal hepatic glucose production and increased insulin release after oral and intravenous glucose loads. Diabetologia 52:2122-2129

24. Jørgensen T, Borch-Johnsen K, Thomsen TF, Ibsen H, Glumer C, Pisinger C (2003) A randomized non-pharmacological intervention study for prevention of ischaemic heart disease: Baseline results Inter99 (1). Eur J Cardiovasc Prev Rehab 10:377-386

25. Grarup N, Andersen G, Krarup NT et al (2008) Association testing of novel type 2 diabetes risk alleles in the JAZF1, CDC123/CAMK1D, TSPAN8, THADA, ADAMTS9, and NOTCH2 loci with insulin release, insulin sensitivity, and obesity in a population-based sample of 4,516 glucose-tolerant middleaged Danes. Diabetes 57:2534-2540

26. Sluiter WJ, Erkelens DW, Reitsma WD, Doorenbos H (1976) Glucose tolerance and insulin release, a mathematical approach I. Assay of the beta-cell response after oral glucose loading. Diabetes 25:241-244

27. Hansen T, Drivsholm T, Urhammer SA et al (2007) The BIGTT test: a novel test for simultaneous measurement of pancreatic $\beta$ cell function, insulin sensitivity, and glucose tolerance. Diabetes Care 30:257-262

28. Freathy RM, Timpson NJ, Lawlor DA et al (2008) Common variation in the FTO gene alters diabetes-related metabolic traits to the extent expected given its effect on BMI. Diabetes $57: 1419-1426$ 
29. Kahn SE, Prigeon RL, McCulloch DK et al (1993) Quantification of the relationship between insulin sensitivity and beta-cell function in human subjects. Evidence for a hyperbolic function. Diabetes 42:1663-1672

30. Rosengren AH, Jokubka R, Tojjar D et al (2010) Overexpression of alpha2A-adrenergic receptors contributes to type 2 diabetes. Science 327:217-220
31. Saxena R, Hivert MF, Langenberg C et al (2010) Genetic variation in GIPR influences the glucose and insulin responses to an oral glucose challenge. Nat Genet 42:142-148

32. Poulsen P, Levin K, Petersen I, Christensen K, Beck-Nielsen H, Vaag A (2005) Heritability of insulin secretion, peripheral and hepatic insulin action, and intracellular glucose partitioning in young and old Danish twins. Diabetes 54:275-283 